\title{
Forecasting energy demand using neural-network-based grey residual modification models
}

\author{
Yi-Chung $\mathrm{Hu}^{1,2 *}$ and Peng Jiang ${ }^{2}$ \\ ${ }^{1}$ College of Management \& College of Tourism, Fujian Agriculture and Forestry University, Fuzhou City, China; \\ and ${ }^{2}$ Department of Business Administration, Chung Yuan Christian University, Chung Li Dist., Taoyuan City, \\ Taiwan
}

Grey forecasting models have taken an important role for forecasting energy demand, particularly the $\operatorname{GM}(1,1)$ model, because they are able to construct a forecasting model using a limited samples without statistical assumptions. To improve prediction accuracy of a $\operatorname{GM}(1,1)$ model, its predicted values are often adjusted by establishing a residual GM(1,1) model, which together form a grey residual modification model. Two main issues should be considered: the sign estimation for a predicted residual and the way the two models are constructed. Previous studies have concentrated on the former issue. However, since both models are usually established in the traditional manner, which is dependent on a specific parameter that is not easily determined, this paper focuses on the latter issue, incorporating the neural-network-based GM(1,1) model into a residual modification model to resolve the drawback. Prediction accuracies of the proposed neural-network-based prediction models were verified using real power and energy demand cases. Experimental results verify that the proposed prediction models perform well in comparison with original ones.

Journal of the Operational Research Society (2017) 68(5), 556-565. doi:10.1057/s41274-016-0130-2;

published online 16 November 2016

Keywords: energy demand; forecasting; grey theory; neural network; residual model

\section{Introduction}

Rapid economic development and ongoing industrialization has led to enormous energy consumption. Energy demand prediction has become increasingly important when devising development plans for a country, and particularly for developing countries (Pi et al, 2010), such as China. Meanwhile, energy management is an important issue for future economic prosperity and environmental security (Suganthi and Samuel, 2012). China has become increasingly influential in energy production and consumption (Liu, 2015). China energy has been mainly provided by coal and crude oil, comprising $2 / 3$ (66\%) and $18 \%$, respectively, of total energy consumed (National Bureau of Statistics of China, 2014). In the past decade, annual energy consumption increases were larger than energy production increases, and China faces not only inevitable environmental impacts, but also the challenge of devising an energy policy forecasting energy demand. Also, energy demand forecasting for China has been an interesting issue (Suganthi and Samuel, 2012).

Grey prediction models are capable of characterizing an unknown system using small data sets (Deng, 1982),

*Correspondence: Yi-Chung Hu, Department of Business Administration, Chung Yuan Christian University, Chung Li Dist., Taoyuan City, Taiwan. E-mail: ychu@cycu.edu.tw without requiring conformance with statistical assumptions. Only few sample data points are required to achieve reliable and acceptable prediction accuracy (Wen, 2004; Wang and Hsu, 2008) and have been widely applied to management, economics, and engineering (e.g. Feng et al, 2012; Chang et al, 2015; Pi et al, 2010; Lee and Tong, 2011; Mao and Chirwa, 2006; Zeng et al, 2016; Chang et al, 2015; Tsaur and Liao, 2007; Wen, 2004; Cui et al, 2013; Wei et al, 2015). Grey prediction systems are particularly appropriate for energy demand forecasting, because energy consumption data are often few and/or do not conform to the usual statistical assumptions, such as normal distribution (Lee and Tong, 2011; Suganthi and Samuel, 2012). Wu et al, (2013) also used practical numerical examples to demonstrate that the small sample usually has more accuracy than the large sample for setting up a grey prediction model. Artificial intelligence techniques, multivariate regression, and time series models (e.g. Ediger and Akar, 2007; Gonzalez and Zamarreno, 2005; Tutun et al, 2015; Lauret et al, 2008; Duran, 2009; Xia et al, 2010) require large sample sizes to achieve reasonable forecasting accuracy (Wang and Hsu, 2008; Feng et al, 2012; Chang et al, 2015; Pi et al, 2010), which is impractical for energy demand forecasting.

The $\operatorname{GM}(1,1)$ model is one of the most frequently used grey prediction models for time series forecasting (Liu and Lin, 
2006). To improve prediction accuracy of the original GM(1,1) model, several improved versions have been proposed, such as a discrete forecasting model (Xie and Liu, 2009), a grey Lotka-Volterra model (Wu et al, 2012), a new model with the fractional order accumulation ( $\mathrm{Wu}$ et al, 2013), a novel $\operatorname{BGM}(1,1)$ using a box plot to analyse data features (Chang et al, 2015), an improved grey model with convolution integral $\operatorname{GMC}(1, n)$ (Wang and Hao, 2016), and a self-adaptive intelligence model (Zeng et al, 2016). Besides, the residual model has been eye-catching and played an important role in grey prediction (Liu and Lin, 2006; Deng, 1982). The residual modification model thus becomes the focus of this study.

When the corresponding residual model is established, predicted values from the original model can be adjusted by those from the residual model. The two models comprise a grey residual modification model, and both models are usually constructed in the same way as the traditional $\operatorname{GM}(1,1)$ model. However, the background value has an important role for the traditional model, but is not easily determined. This leads to some well-known prediction models using the traditional $\operatorname{GM}(1,1)$ model, developed for residual sign estimation to improve the prediction accuracy of the residual modification model, which also encounter the difficulty in determining background value, for example the MLP-GM $(1,1)$ model based on multi-layer perceptron (MLP) (Hsu and Chen, 2003) and the GP-GM(1,1) model based on genetic programming (GP; Lee and Tong, 2011). The neuralnetwork-based GM(1,1) (NN-GM(1,1)) model is free of the dependency on the background value (Hu et al, 2001) and performs well in comparison with the traditional $\operatorname{GM}(1,1)$ model. Therefore, it is interesting to investigate the impact on prediction accuracy of the proposed neural-network-based residual modification models on energy demand forecasting for China by using the NN-GM(1,1) model rather than the traditional $\operatorname{GM}(1,1)$ model.

The remainder of the paper is organized as follows. Section 2 introduces the traditional grey residual modification model, and Section 3 introduces the NN-GM $(1,1)$ model and the proposed neural-network-based residual modification model. On the basis of the MLP-GM $(1,1)$ and GP-GM $(1,1)$ models, Section 4 examines the forecasting performances of the proposed prediction models using real cases of power and energy demand. Section 5 discusses the outcomes and presents conclusions.

\section{Traditional grey residual modification model}

\subsection{Traditional $G M(1,1)$ model}

The computational steps to construct a traditional $\operatorname{GM}(1,1)$ model are as follows:
Step 1 Present an original and nonnegative data sequence $\mathbf{x}^{(0)}=\left(x_{1}^{(0)}, x_{2}^{(0)}, \ldots, x_{n}^{(0)}\right)$, provided by one system and consisting of $n$ samples.

Step 2 Perform the accumulated generating operation (AGO).

Identify the potential regularity hidden in $\mathbf{x}^{(0)}$ using AGO (Liu and Lin, 2006; Duran, 2009) to generate a new sequence, $\mathbf{x}^{(1)}=\left(x_{1}^{(1)}, x_{2}^{(1)}, \ldots, x_{n}^{(1)}\right)$,

$$
x_{k}^{(1)}=\sum_{j=1}^{k} x_{k}^{(0)}, \quad k=1,2, \ldots, n
$$

and $x_{1}^{(1)}, x_{2}^{(1)}, \ldots, x_{n}^{(1)}$ can be then approximated by a first-order differential equation,

$$
\frac{d \mathbf{x}^{(1)}}{d t}+a x^{(1)}=b
$$

where $a$ and $b$ are the developing coefficient and control variable, respectively. The predicted value, $\hat{x}_{k}^{(1)}$, for $x_{k}^{(1)}$ can be obtained by solving the differential equation with initial condition $x_{1}^{(1)}=x_{1}^{(0)}$ :

$$
\hat{x}_{k}^{(1)}=\left(x_{1}^{(0)}-\frac{b}{a}\right) e^{-a(k-1)}+\frac{b}{a}
$$

Step 3 Determine the developing coefficient and control variable.

$a$ and $b$ can be obtained using the ordinary leastsquares method:

$$
[a, b]^{T}=\left(\mathbf{B}^{T} \mathbf{B}\right)^{-1} \mathbf{B}^{T} \mathbf{y}
$$

where

$$
\begin{gathered}
\mathbf{B}=\left[\begin{array}{cc}
-z_{2}^{(1)} & 1 \\
-z_{3}^{(1)} & 1 \\
\vdots & \vdots \\
-z_{n}^{(1)} & 1
\end{array}\right] \\
z_{k}^{(1)}=\alpha x_{k}^{(1)}+(1-\alpha) x_{k}^{(1)} \\
\mathbf{y}=\left[x_{2}^{(0)}, x_{3}^{(0)}, \ldots x_{n}^{(0)}\right]^{T}
\end{gathered}
$$

where $z_{k}^{(1)}$ is the background value. $\alpha$ is usually specified as 0.5 for convenience, but this is not an optimal setting. Thus, $a$ and $b$ are fully dependent on $z_{k}^{(1)}$, which is not easily determined. 
Step 4 Perform the inverse accumulated generating operation (IAGO).

Using the IAGO, the predicted value of $x_{k}^{(0)}$ is

$$
\hat{x}_{k}^{(0)}=\hat{x}_{k}^{(1)}-\hat{x}_{k-1}^{(1)}, \quad k=2,3, \ldots, n
$$

Therefore,

$$
\hat{x}_{k}^{(0)}=\left(1-e^{a}\right)\left(x_{1}^{(0)}-\frac{b}{a}\right) e^{-a(k-1)}, \quad k=2,3, \ldots, n
$$

and note that $\hat{x}_{1}^{(1)}=\hat{x}_{1}^{(0)}$ holds.

\subsection{Residual modification using traditional $\operatorname{GM}(1,1)$ models}

A residual modification model is usually established using traditional GM(1,1) models. The computational steps of constructing a traditional residual modification model are as follows:

Step 1 Establish a traditional GM(1,1) model for $\mathbf{x}^{(0)}$.

Step 2 Generate the sequence of absolute residual values, $\boldsymbol{\varepsilon}^{(0)}=\left(\varepsilon_{2}^{(0)}, \varepsilon_{3}^{(0)}, \ldots, \varepsilon_{n}^{(0)}\right)$, where

$$
\varepsilon_{k}^{(0)}=\left|x_{k}^{(0)}-\hat{x}_{k}^{(0)}\right|, \quad k=2,3, \ldots, n
$$

Step 3 Establish a residual model.

A residual model is established as a traditional $\operatorname{GM}(1,1)$ model for $\boldsymbol{\varepsilon}^{(0)}$. Similar to $\hat{x}_{k}^{(0)}$, the predicted residual of $\varepsilon_{k}^{(0)}$ is

$$
\begin{aligned}
\hat{\varepsilon}_{k}^{(0)} & =\left(1-e^{a_{\varepsilon}}\right)\left(\varepsilon_{2}^{(0)}-\frac{b_{\varepsilon}}{a_{\varepsilon}}\right) e^{-a_{\varepsilon}(k-1)}, \\
k & =3,4, \ldots, n
\end{aligned}
$$

where $a_{\varepsilon}$ and $b_{\varepsilon}$ are the developing coefficient and the control variable, respectively, and are also fully dependent on the background value.

Step 4 Perform residual modification.

A predicted value $\hat{x}_{k^{t r}}^{(0)}$ can be obtained by adding or subtracting $\hat{\varepsilon}_{k}^{(0)}$ from original $\hat{x}_{k}^{(0)}$ (Hsu and Wen, 1998).

$$
\hat{x}_{k^{t r}}^{(0)}=\hat{x}_{k}^{(0)}+s_{k} \hat{\varepsilon}_{k}^{(0)}, \quad k=2,3, \ldots, n
$$

where $s_{k}$ denotes the positive or negative sign for $\hat{\varepsilon}_{k}^{(0)}$. The determination of $s_{k}$ can be dependent on the mechanism of sign estimation provided by other residual modification models, for instance the MLP$\operatorname{GM}(1,1)$ and GP-GM(1,1) models. For simplicity, the sign estimation methods of those two prediction models are omitted.

\section{Neural-network-based grey residual modification model}

\subsection{NN-GM(1,1) model}

Because $z_{k}^{(1)}$ is not easily determined, it is quite reasonable to consider finding $a$ and $b$ without requiring $z_{k}^{(1)}$. A cost function $E(a, b)$,

$$
E(a, b)=\frac{1}{2} \sum_{k}\left(x_{k}^{(0)}-\hat{x}_{k}^{(0)}\right)^{2}, \quad k=2,3, \ldots, n
$$

was built for the NN-GM(1,1) model, where $a$ and $b$ are the connection weights. The model itself was a widely used single-layer perceptron (SLP). Similar to the back-propagation algorithm (BP; Smith and Gupta, 2002), the computational steps to constructing such a model are as follows:

Step 1 Present a randomly selected sequence $(k, 1,1)$ $(k=2,3, \ldots, n)$ with $x_{k}^{(0)}$ as its desired output to NN-GM(1,1).

Step 2 Calculate the actual output $\hat{x}_{k}^{(0)}$ of NN-GM(1,1).

Step 3 Adjust the connection weights. For $(k, 1,1), a$ and $b$ are adjusted to $a+\Delta a$ and $b+\Delta b$, respectively. Then $\Delta a$ and $\Delta b$ can be derived by the gradient descent method on the cost function, and

$$
\begin{gathered}
\Delta a=\eta\left(x_{k}^{(0)}-\hat{x}_{k}^{(0)}\right) V_{a k} \\
\Delta b=\eta\left(x_{k}^{(0)}-\hat{x}_{k}^{(0)}\right) V_{b k}
\end{gathered}
$$

where

$$
\begin{gathered}
V_{a k}=\left[\left(-e^{a}\right)\left(x_{1}^{(0)}-\frac{b}{a}\right) e^{-a(k-1)}+\left(1-e^{a}\right)\left(\frac{b}{a^{2}}\right)\right. \\
\left.e^{-a(k-1)}+\left(1-e^{a}\right)\left(x_{1}^{(0)}-\frac{b}{a}\right)(-k+1) e^{-a(k-1)}\right] \\
V_{b k}=\left[\left(1-e^{a}\right)\left(-\frac{1}{a}\right) e^{-a(k-1)}\right]
\end{gathered}
$$

Step 4 Terminate when a pre-specified number of iterations have been performed; otherwise, return to Step 1.

\subsection{Residual modification using NN-GM(1,1) models}

In the proposed neural-network-based model, traditional $\operatorname{GM}(1,1)$ models are no longer used; rather, $\operatorname{NN}-\mathrm{GM}(1,1)$ models considered. The construction of the proposed grey prediction model is described as follows:

Step 1 Establish a NN-GM(1,1) model for $\mathbf{x}^{(0)}$. 
Step 2 For $\mathbf{x}^{(0)}$, generate the sequence of absolute residual values, $\boldsymbol{\varepsilon}^{(0)}=\left(\varepsilon_{2}^{(0)}, \varepsilon_{3}^{(0)}, \ldots, \varepsilon_{n}^{(0)}\right)$.

Step 3 Establish a residual NN-GM(1,1) model.

A residual NN-GM $(1,1)$ model, using all sequences of absolute residual values, is established where $a_{\varepsilon}$ and $b_{\varepsilon}$ are connection weights of a SLP for processing residuals. $\Delta a_{\varepsilon}$ and $\Delta b_{\varepsilon}$ may be derived with respect to $a_{\varepsilon}$ and $b_{\varepsilon}$, respectively, by defining a cost function

$$
E\left(a_{\varepsilon}, b_{\varepsilon}\right)=\frac{1}{2} \sum_{k}\left(\varepsilon_{k}^{(0)}-\hat{\varepsilon}_{k}^{(0)}\right)^{2}, \quad k=3,4, \ldots, n
$$

Similar to $a$ and $b, a_{\varepsilon}$ and $b_{\varepsilon}$ can be adjusted to $a_{\varepsilon}+\Delta a_{\varepsilon}$ and $b_{\varepsilon}+\Delta b_{\varepsilon}$, respectively, after presenting a randomly selected sequence $(k, 1,1)(k=3,4, \ldots$, $n)$ with desired output $\varepsilon_{k}^{(0)}$ to the SLP related to, where

$$
\begin{aligned}
& \Delta a_{\varepsilon}=\eta\left(\varepsilon_{k}^{(0)}-\hat{\varepsilon}_{k}^{(0)}\right) V_{a k} \\
& \Delta b_{\varepsilon}=\eta\left(\varepsilon_{k}^{(0)}-\hat{\varepsilon}_{k}^{(0)}\right) V_{b k}
\end{aligned}
$$

and

$$
\begin{aligned}
V_{a k}= & \left(-e^{a}\right)\left(\varepsilon_{2}^{(0)}-\frac{b}{a}\right) e^{-a(k-1)} \\
& +\left(1-e^{a}\right)\left(\frac{b}{a^{2}}\right) e^{-a(k-1)} \\
& +\left(1-e^{a}\right)\left(\varepsilon_{2}^{(0)}-\frac{b}{a}\right)(-k+1) e^{-a(k-1)} \\
V_{b k}= & \left(1-e^{a}\right)\left(-\frac{1}{a}\right) e^{-a(k-1)}
\end{aligned}
$$

Step 4 Perform residual modification.

A predicted value $\hat{x}_{k^{n n r}}^{(0)}$ is produced by adding or subtracting $\hat{\varepsilon}_{k}^{(0)}$ from the original $\hat{x}_{k}^{(0)}$,

$$
\hat{x}_{k^{n n r}}^{(0)}=\hat{x}_{k}^{(0)}+s_{k} \hat{\varepsilon}_{k}^{(0)}, \quad k=2,3, \ldots, n
$$

As demonstrated in Figure 1, two independent SLPs were employed to establish the proposed neural-network-based grey residual modification model: one each for the original and residual sequences. The $\operatorname{MLP}-\operatorname{GM}(1,1)$ and $\operatorname{GP}-\operatorname{GM}(1,1)$ models used two traditional $\operatorname{GM}(1,1)$ models, independently, and artificial intelligence tools were applied to effectively determine $s_{k}$. The flow chart of the proposed residual modification model is illustrated in Figure 2.

When the NN-GM(1,1) models were incorporated into the MLP-GM(1,1) and GP-GM(1,1) models, rather traditional
GM(1,1) models, two new prediction models, NN-MLP$\operatorname{GM}(1,1)$ and NN-GP-GM(1,1), were able to remove the requirement of determining background values.

\section{Empirical results}

Empirical studies were conducted using real data sets to compare energy demand forecasting ability of the proposed NN-MLP-GM(1,1) and NN-GP-GM(1,1) models against original $\operatorname{GM}(1,1)$, NN-GM(1,1), MLP-GM(1,1), and NN-GPGM(1,1) models. Mean absolute percentage error (MAPE) was employed to measure prediction performance, as this can be treated as the benchmark and is more stable than the commonly used mean absolute error and root mean square error (Makridakis, 1993; Lee and Shih, 2011). MAPE with respect to $x_{k}^{(0)}$ is

$$
\mathrm{MAPE}=\sum_{k \in T} \frac{e_{k}}{|T|}
$$

where $T$ denotes the set of training or test data, whereas $e_{k}$ is the absolute percentage error (APE) with respect to $x_{k}^{(0)}$,

$$
e_{k}=\frac{\left|x_{k}^{(0)}-\hat{x}_{k^{p}}^{(0)}\right|}{x_{k}^{(0)}} \times 100 \%
$$

where $\hat{x}_{k^{p}}^{(0)}$ is a predicted value (e.g. $\left.\hat{x}_{k}^{(0)}, \hat{x}_{k^{t r}}^{(0)}, \hat{x}_{k^{m r r}}^{(0)}\right)$ with respect to $x_{k}^{(0)}$. Lewis (1982) proposed MAPE criteria for evaluating a forecasting model, where MAPE $\leq 10,10<\mathrm{MAPE} \leq 20$, $20<$ MAPE $\leq 50$, and MAPE $>50$ correspond to high, good, reasonable, and weak forecasting models, respectively.

\subsection{Applications to energy demand forecasting}

4.1.1. Case I An experiment was conducted on the historical annual power demand of Taiwan from 1985 to 2000. As in Hsu and Chen (2003), data from 1985 to 1998 were reserved for the model-fitting, and data from 1999 to 2000 were used for ex post testing. Table 1 summarizes forecasting results, reported by Hsu and Chen (2003), of original $\operatorname{GM}(1,1)$ and MLP$\operatorname{GM}(1,1)$ models, along with the corresponding details for the proposed NN-MLP-GM $(1,1)$ model. From Table 1, we can see that the MAPE of the original $\operatorname{GM}(1,1)$, the $\operatorname{MLP}-\operatorname{GM}(1,1)$, and the NN-MLP-GM(1,1) models for model-fitting was 1.54 , 0.57 , and $1.56 \%$, respectively. And for ex post testing, the MAPE was $3.88,1.29$, and $0.78 \%$, respectively.

It is noteworthy that, although the NN-MLP-GM $(1,1)$ model is slightly inferior to the original $\operatorname{GM}(1,1)$ and the MLP$\operatorname{GM}(1,1)$ models for model-fitting, it is superior to the original $\operatorname{GM}(1,1)$ and the MLP-GM(1,1) models for ex post testing. Actually, when evaluating a prediction model, more emphasis should be placed on generalization rather than model-fitting (Luo et al, 2013). In this case, the MLP-GM(1,1) model seems 


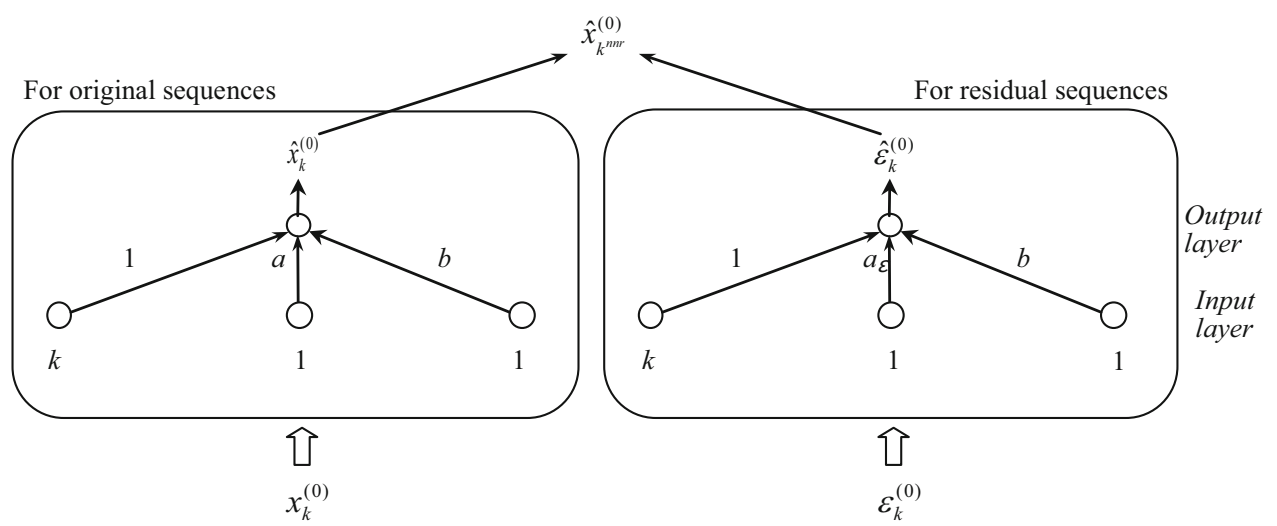

Figure 1 A neural-network-based residual modification model.

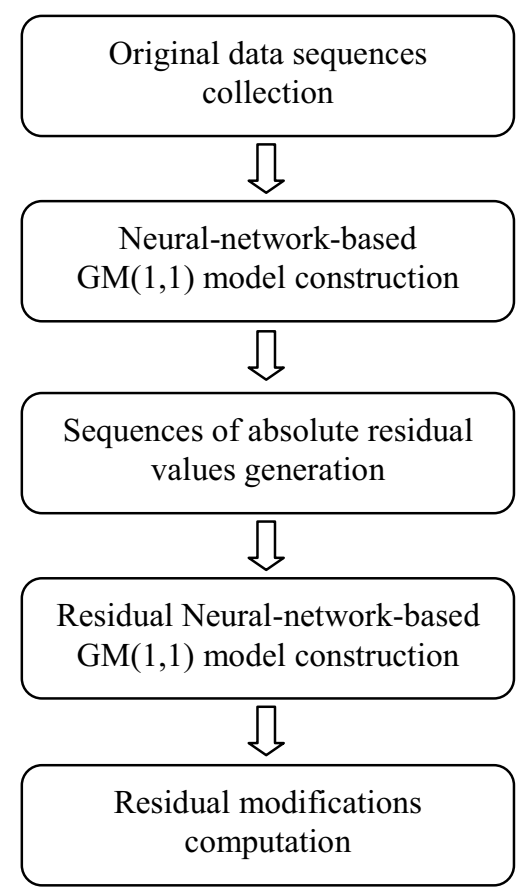

Figure 2 Flow chart of the proposed residual modification model.

to suffer from over-fitting. Figure 3 demonstrates the superiority of the generalization ability of the proposed NN-MLP$\operatorname{GM}(1,1)$ model over the original $\operatorname{GM}(1,1)$ and the MLPGM(1,1) models.

4.1.2. Case II The second experiment was conducted on the historical annual energy demand of China, collected from 1990 to 2007. Same as Lee and Tong (2011), data from 1990 to 2003 were used for the model-fitting, and data from 2004 to 2007 were used for ex post testing. Forecasting results from Lee and Tong (2011) obtained by original GM(1,1), MLPGM(1,1) and GP-GM(1,1) models are summarized in Table 2, along with the corresponding details for the proposed $\mathrm{NN}$ -
MLP-GM(1,1) and NN-GP-GM(1,1) models. Table 2 shows that the MAPE of the original $\operatorname{GM}(1,1)$, the $\operatorname{MLP}-\operatorname{GM}(1,1)$, the $\operatorname{GP}-\mathrm{GM}(1,1)$, the NN-GM(1,1), the NN-MLP-GM(1,1), and the NN-GP-GM(1,1) models for model-fitting was 4.13, 3.61, $2.59,3.81,4.15$, and $2.80 \%$, respectively. And for ex post testing, the MAPE was 26.21, 20.23, 20.23, 28.71, 14.81, and $14.81 \%$, respectively. Since a change on an epic scale happened to 2004, this can explain why results of the ex post testing is not as good as those of the model-fitting.

Similar to Case I, although MAPE obtained by NN-MLP$\operatorname{GM}(1,1)$ and NN-GP-GM(1,1) models is slightly inferior to that from MLP-GM(1,1) and GP-GM(1,1), respectively, for model-fitting, they are superior to $\operatorname{MLP}-\mathrm{GM}(1,1)$ and GP$\operatorname{GM}(1,1)$, respectively, for ex post testing. In this case, it seems that both MLP-GM(1,1) and GP-GM(1,1) models suffer from over-fitting. The predicted values obtained by different forecasting models are illustrated in Figure 4. The proposed NN-MLP-GM(1,1) and NN-GP-GM(1,1) models show "good" generalization ability, whereas the other prediction models have only "reasonable" forecasting ability for testing data. The generalization ability of the NN-MLP-GM(1,1) and the NN-GP-GM $(1,1)$ models are conspicuous.

4.1.3. Case III The third experiment was conducted on historical annual electricity demand of China, collected from China Statistical Yearbook (National Bureau of Statistics of China, 2014), 1981-2002. Following (Zhou et al, 2006), data from 1981 to 1998 were used for model-fitting, and from 1999 to 2002 for ex post testing. The forecasting results obtained from the different forecasting models are summarized in Table 3. All the models have "high" forecasting ability on the training and test data.

Table 3 shows that results obtained by the proposed NNMLP-GM(1,1) and NN-GP-GM(1,1) models are satisfactory. The MAPE of the original $\operatorname{GM}(1,1)$, the MLP-GM(1,1), the $\operatorname{GP}-\mathrm{GM}(1,1)$, the NN-GM(1,1), the NN-MLP-GM(1,1), and the NN-GP-GM(1,1) models for model-fitting was 2.28, 2.03, $1.44,1.84,1.84$, and $1.28 \%$, respectively. And for ex post 
Table 1 Prediction accuracy obtained by different forecasting models for power demand (unit: $10^{3} \mathrm{Wh}$ )

\begin{tabular}{|c|c|c|c|c|c|c|c|}
\hline \multirow[t]{2}{*}{ Year } & \multirow[t]{2}{*}{ Actual } & \multicolumn{2}{|c|}{ Original $\operatorname{GM}(1,1)$} & \multicolumn{2}{|c|}{$M L P-G M(1,1)$} & \multicolumn{2}{|c|}{$N N-M L P-G M(1,1)$} \\
\hline & & Predicted & $A P E$ & Predicted & $A P E$ & Predicted & $A P E$ \\
\hline 1985 & 47919102 & 47919102 & 0 & 47919102 & 0 & 47919102 & 0 \\
\hline 1986 & 53812862 & 56318092 & 4.66 & 53812862 & 0 & 53812862 & 0 \\
\hline 1987 & 59174751 & 60319829 & 1.94 & 59630904 & 0.77 & 63072052.4 & 6.59 \\
\hline 1988 & 65227727 & 64605914 & 0.95 & 65310510 & 0.13 & 67141427.8 & 2.93 \\
\hline 1989 & 69251809 & 69196550 & 0.08 & 69917174 & 0.96 & 71470372.97 & 3.20 \\
\hline 1990 & 74344947 & 74113379 & 0.31 & 74850394 & 0.68 & 76076268.3 & 2.33 \\
\hline 1991 & 80977405 & 79379577 & 1.97 & 80133358 & 1.04 & 80977428.0 & 0 \\
\hline 1992 & 85290354 & 85019971 & 0.32 & 85790897 & 0.59 & 86193211.8 & 1.059 \\
\hline 1993 & 92084684 & 91061148 & 1.11 & 91849611 & 0.26 & 91751490.1 & 0.36 \\
\hline 1994 & 98561004 & 97531587 & 1.04 & 98337985 & 0.23 & 97657263.9 & 0.92 \\
\hline 1995 & 105368193 & 104461790 & 0.86 & 105286530 & 0.08 & 103943602.7 & 1.35 \\
\hline 1996 & 111139816 & 111884424 & 0.67 & 111040924 & 0.09 & 110634911.7 & 0.45 \\
\hline 1997 & 118299046 & 119834482 & 1.30 & 118971794 & 0.57 & 117757193.7 & 0.46 \\
\hline 1998 & 128129801 & 128349438 & 0.17 & 127467127 & 0.52 & 125338144.8 & 2.18 \\
\hline MAPE & & & 1.54 & & 0.57 & & 1.56 \\
\hline 1999 & 131725892 & 137469433 & 4.36 & 133459644 & 1.32 & 133406202.6 & 1.28 \\
\hline 2000 & 142412887 & 147237458 & 3.39 & 144204700 & 1.26 & 141995935.9 & 0.29 \\
\hline MAPE & & & 3.88 & & 1.29 & & 0.78 \\
\hline
\end{tabular}

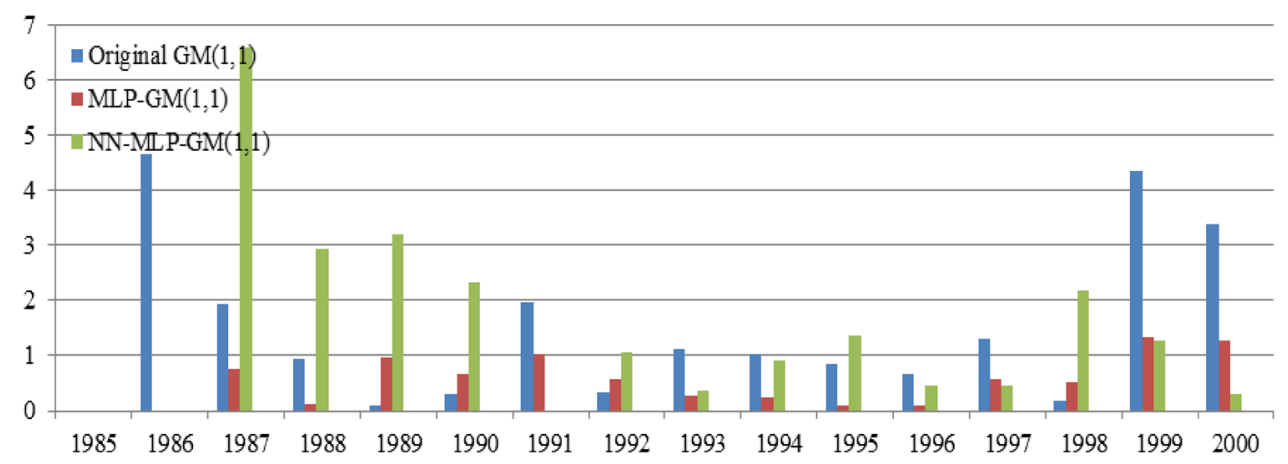

Figure 3 Absolute percentage errors by different prediction models for Case I.

testing, the MAPE was $7.24,3.90,3.90,10.35,3.34$, and $3.34 \%$, respectively. The proposed NN-MLP-GM(1,1) and NN-GP-GM(1,1) models have superior fitting and generalization ability compared to $\operatorname{MLP}-\operatorname{GM}(1,1)$ and $\operatorname{GP}-\operatorname{GM}(1,1)$ models, respectively. Figure 5 also demonstrates the superiority of the generalization ability of NN-MLP-GM(1,1) and NN-GP-GM $(1,1)$ models over the other prediction models.

\section{Discussion and conclusions}

Energy demand forecasting can be regarded as a grey system problem (Pi et al, 2010; Suganthi and Samuel, 2012) because several factors, such as income and population, influence energy demand but the precise relationships are not clear. That is, although relationships exist between input factors and dependent variable in the real problems, but it is not distinct about what these relationships are (Hu, 2016; $\mathrm{Hu}$ et al, 2015).
Energy demand data are often limited and do not conform to the usual statistical assumptions, such as normal distribution. The $\operatorname{GM}(1,1)$ model is the most frequently used grey prediction model and has played an important role in energy demand prediction because it requires only limited samples to construct a prediction model without statistical assumptions. However, the traditional residual modification model has suffered from determination of the background value, as does the traditional $\operatorname{GM}(1,1)$ model, whereas the $\operatorname{NN}-\operatorname{GM}(1,1)$ model is able to directly determine the developing coefficient and control variable using a SLP without requiring the background value. The NN-GM(1,1) model is also simple to implement as a computer program. Therefore, it is reasonable to replace the traditional $\operatorname{GM}(1,1)$ model with the $\mathrm{NN}$ $\operatorname{GM}(1,1)$ model for a grey residual modification model. It is noted that, unlike the traditional SLP, the NN-GM(1,1) model does not use the sigmoid function as its activation function. 


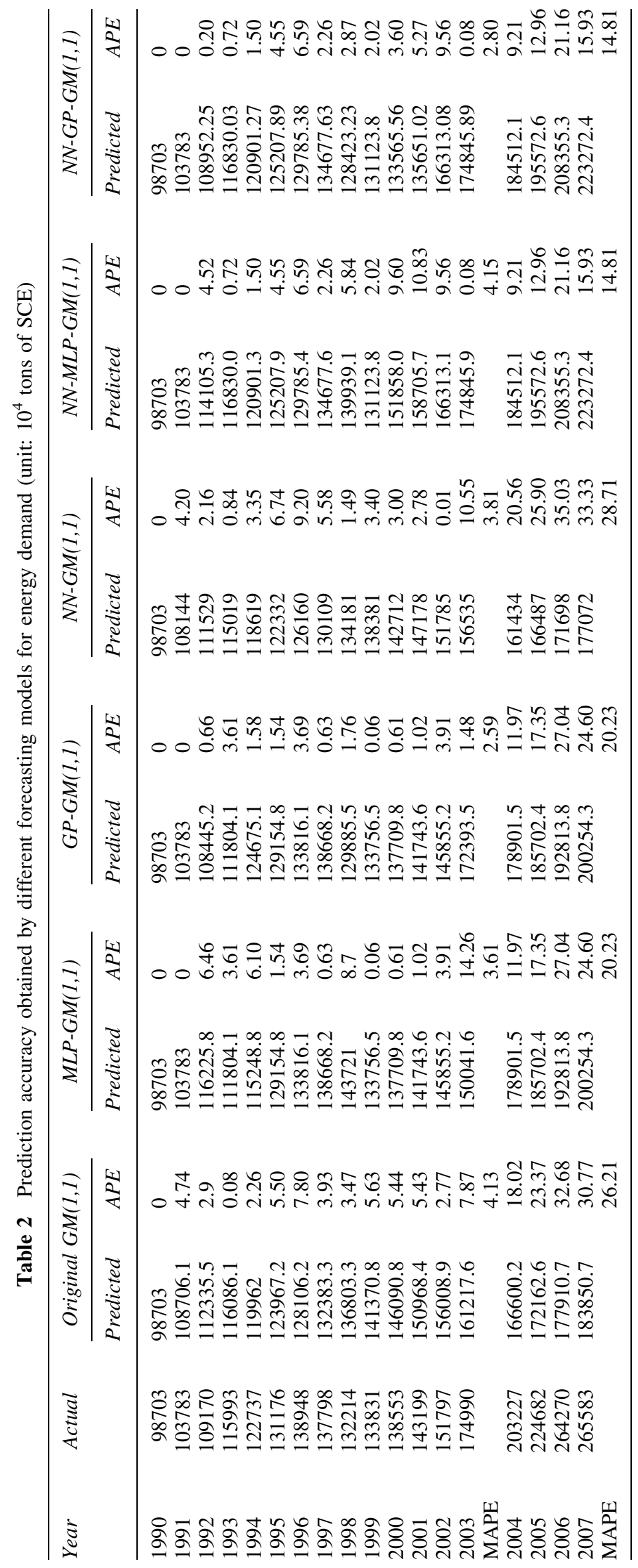




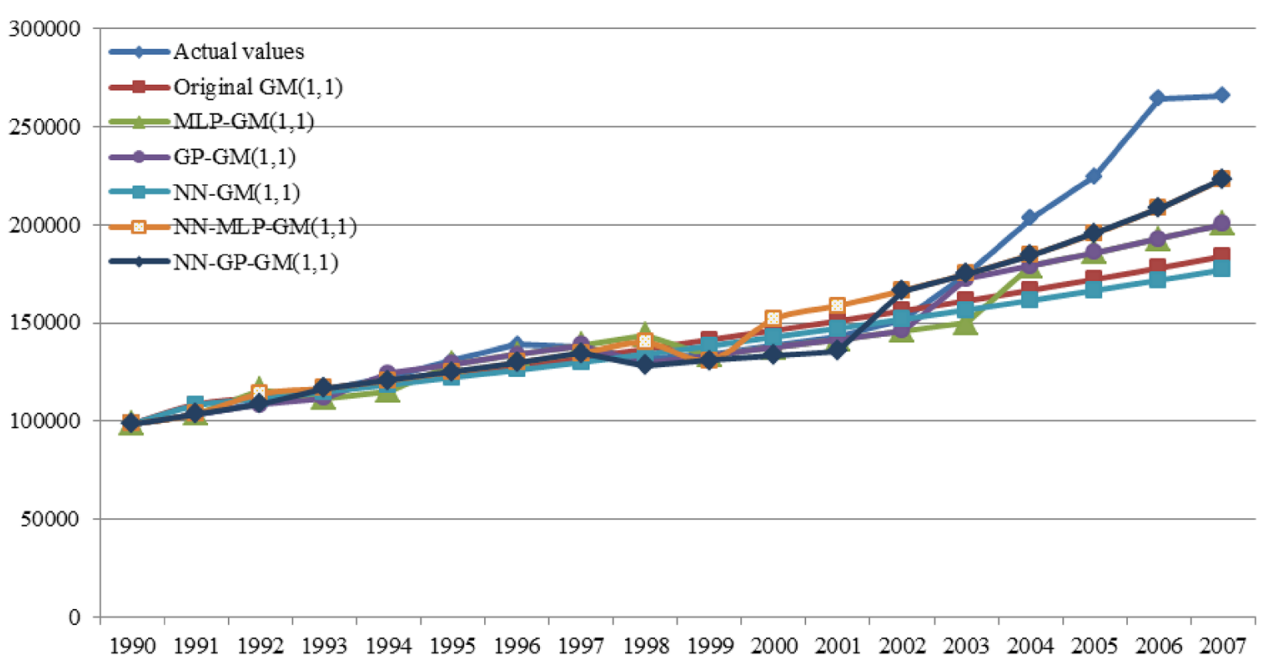

Figure 4 Predicted values obtained by different prediction models.

Table 3 Prediction accuracy obtained by different forecasting models for electricity demand (unit: $100 \mathrm{million} \mathrm{kWh}$ )

\begin{tabular}{|c|c|c|c|c|c|c|c|c|c|c|c|c|c|}
\hline \multirow[t]{2}{*}{ Year } & \multirow[t]{2}{*}{ Actual } & \multicolumn{2}{|c|}{ Original $\operatorname{GM}(1,1)$} & \multicolumn{2}{|c|}{$M L P-G M(1,1)$} & \multicolumn{2}{|c|}{$G P-G M(1,1)$} & \multicolumn{2}{|c|}{$N N-G M(1,1)$} & \multicolumn{2}{|c|}{$N N-M L P-G M(1,1)$} & \multicolumn{2}{|c|}{$N N-G P-G M(1,1)$} \\
\hline & & Predicted & $A P E$ & Predicted & $A P E$ & Predicted & $A P E$ & Predicted & $A P E$ & Predicted & $A P E$ & Predicted & $A P E$ \\
\hline 1981 & 3096 & 3096 & 0 & 3096 & 0 & 3096 & 0 & 3096 & 0 & 3096 & 0 & 3096 & 0 \\
\hline 1982 & 3280 & 3327.7 & 1.45 & 3280 & 0 & 3280 & 0 & 3230.1 & 1.52 & 3280 & 0 & 3280 & 0 \\
\hline 1983 & 3519 & 3611.5 & 2.63 & 3638.0 & 3.38 & 3585.0 & 1.88 & 3516.7 & 0.07 & 3551.4 & 0.92 & 3551.4 & 0.92 \\
\hline 1984 & 3778 & 3919.5 & 3.75 & 3888.3 & 2.92 & 3888.3 & 2.92 & 3828.7 & 1.34 & 3804.6 & 0.70 & 3804.6 & 0.70 \\
\hline 1985 & 4118 & 4253.9 & 3.30 & 4217.1 & 2.41 & 4217.1 & 2.41 & 4168.4 & 1.22 & 4138.1 & 0.49 & 4138.1 & 0.49 \\
\hline 1986 & 4507 & 4616.7 & 2.43 & 4573.3 & 1.47 & 4573.3 & 1.47 & 4538.3 & 0.69 & 4500.3 & 0.15 & 4500.3 & 0.15 \\
\hline 1987 & 4985 & 5010.5 & 0.51 & 4959.4 & 0.51 & 4959.4 & 0.51 & 4940.9 & 0.88 & 4893.4 & 1.84 & 4988.5 & 0.07 \\
\hline 1988 & 5467 & 5437.9 & 0.53 & 5377.6 & 1.63 & 5498.2 & 0.57 & 5379.3 & 1.60 & 5438.9 & 0.51 & 5438.9 & 0.51 \\
\hline 1989 & 5865 & 5901.7 & 0.63 & 5830.7 & 0.59 & 5830.7 & 0.59 & 5856.5 & 0.14 & 5931.2 & 1.13 & 5931.2 & 1.13 \\
\hline 1990 & 6230 & 6405.1 & 2.81 & 6321.4 & 1.47 & 6321.4 & 1.47 & 6376.2 & 2.35 & 6469.7 & 3.85 & 64 & 3.85 \\
\hline 1991 & 6775 & 6951.4 & 2.60 & 6852.8 & 1.15 & 6852.8 & 1.15 & 6941.9 & 2.46 & 7059.1 & 4.19 & 6824.7 & 0.73 \\
\hline 1992 & 7542 & 7544.3 & 0.03 & 7428.1 & 1.51 & 7428.1 & 1.51 & 7557.8 & 0.21 & 7411.0 & 1.74 & 7411.0 & 1.74 \\
\hline 1993 & 8426.5 & 8187.8 & 2.83 & 8050.8 & 4.46 & 8324.8 & 1.21 & 8228.3 & 2.35 & 8044.4 & 4.53 & 8044.4 & 4.53 \\
\hline 1994 & 9260.4 & 8886.2 & 4.04 & 8724.8 & 5.78 & 9047.6 & 2.30 & 8958.4 & 3.26 & 9188.9 & 0.77 & 9188.9 & 0.77 \\
\hline 1995 & 10023.4 & 9644.1 & 3.78 & 9834.3 & 1.89 & 9834.3 & 1.89 & 9753.2 & 2.70 & 10042 & 0.19 & 10042 & 0.19 \\
\hline 1996 & 10764.3 & 10466.7 & 2.76 & 10690.9 & 0.68 & 10690.9 & 0.68 & 10618.6 & 1.35 & 10980.5 & 2.01 & 10980.5 & 2.01 \\
\hline 1997 & 11284.4 & 11359.5 & 0.67 & 11623.7 & 3.01 & 11095.3 & 1.68 & 11560.7 & 2.45 & 12014.1 & 6.47 & 11107.2 & 1.57 \\
\hline 1998 & 11598.4 & 12328.4 & 6.29 & 12017.1 & 3.61 & 12017.1 & 3.61 & 12586.4 & 8.52 & 12018.2 & 3.62 & 12018.2 & 3.62 \\
\hline MAPE & & & 2.28 & & 2.03 & & 1.44 & & 1.84 & & 1.84 & & 1.28 \\
\hline 1999 & 12305.2 & 13379.9 & 8.73 & 13013 & 5.75 & 13013.0 & 5.75 & 13703.1 & 11.36 & 12991.2 & 5.57 & 12991.2 & 5.57 \\
\hline 2000 & 13471.4 & 14521.2 & 7.79 & 14088.8 & 4.58 & 14088.8 & 4.58 & 14918.9 & 10.74 & 14026.9 & 4.12 & 14026.9 & 4.12 \\
\hline 2001 & 14633.5 & 15759.8 & 7.70 & 15250.3 & 4.21 & 15250.3 & 4.21 & 16242.5 & 10.99 & 15124.9 & 3.36 & 15124.9 & 3.36 \\
\hline 2002 & 16331.5 & 17104 & 4.73 & 16503.6 & 1.05 & 16503.5 & 1.05 & 17683.6 & 8.279 & 16283.3 & 0.30 & 16283.3 & 0.30 \\
\hline MAPE & & & 7.24 & & 3.90 & & 3.90 & & 10.35 & & 3.34 & & 3.34 \\
\hline
\end{tabular}

Some improved residual modification models, such as MLP-GM(1,1) and GP-GM(1,1), focused on residual sign estimation, but they retain the drawback of the traditional GM(1,1) model. On the other hand, the proposed NN-MLPGM(1,1) and NN-GP-GM $(1,1)$ models were developed from MLP-GM(1,1) and GP-GM(1,1) models, respectively, by substituting $\mathrm{NN}-\mathrm{GM}(1,1)$ for traditional $\mathrm{GM}(1,1)$ models. Therefore, the proposed residual modification model can estimate residual signs effectively and is free from the drawback of the traditional GM(1,1) model.

Real cases of energy demand data from China were used to evaluate the forecasting performances of the proposed NNMLP-GM(1,1) and NN-GP-GM(1,1) models. The outcomes verified that the proposed forecasting models perform well. Zhou et al (2006) showed that for Case III, MAPE for an autoregressive integrated moving average (ARIMA) and 


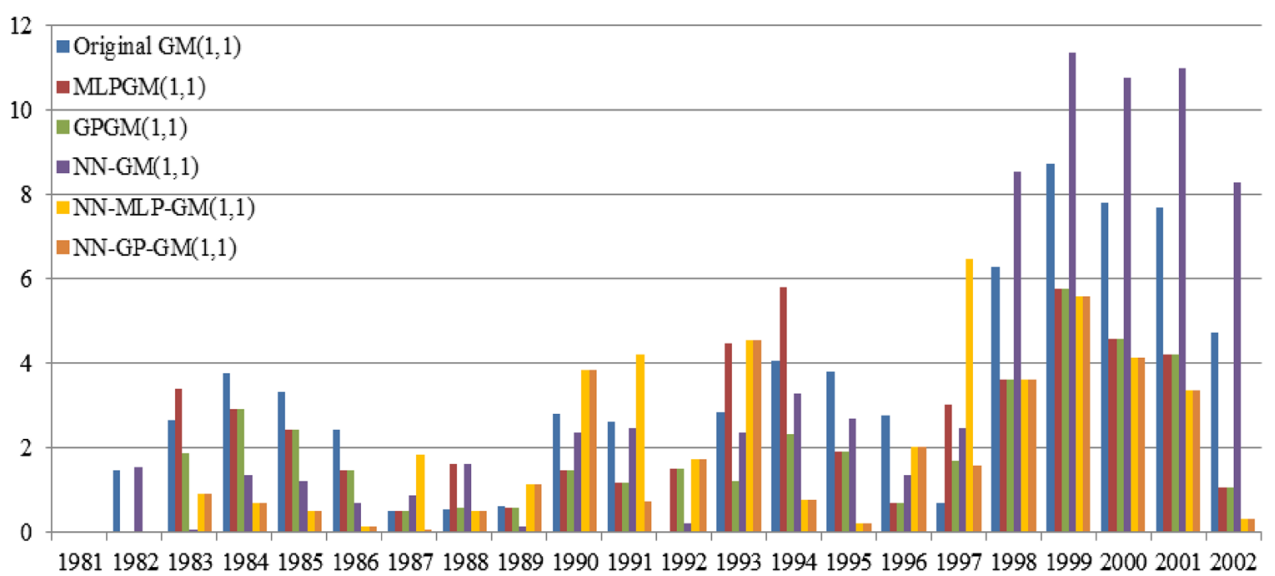

Figure 5 Absolute percentage errors by different prediction models for Case III.

trigonometric grey prediction model was 3.25 and $2.12 \%$, for model-fitting, respectively, which are inferior to the proposed NN-MLP-GM(1,1) and NN-GP-GM(1,1) models. For Cases II and III, it is interesting to note that the NN$\operatorname{GM}(1,1)$ model is superior to the traditional $\operatorname{GM}(1,1)$ model for model-fitting, but inferior for ex post testing. In other words, the NN-GM(1,1) model appears to be over-fitting. Experimental results show that the generalization ability of NN-MLP-GM $(1,1)$ and NN-GP-GM $(1,1)$ models are superior to the MLP-GM(1,1) and GP-GM(1,1) models. Thus, the generalization ability of a residual modification model could be improved by incorporating NN-GM(1,1) models.

The SLP in this study was trained on the basis of the BP using gradient descent. The learning is continued until a convergent condition is reached. It is known that one drawback of using BP is that a local minimum (Weiss and Kulikowski, 1991) is likely to be stuck during the learning process. Therefore, other optimization techniques such as genetic algorithm (GA; Goldberg, 1989; Man et al, 1999) could be applied to automatically determine the connection weights. In parenthesis, in comparison with the BP, an advantage of using GA is that a local minimum is unlikely to be stuck (Rooij et al, 1996; Vonkj et al, 1997; Hu, 2010). Additionally, the MLP-GM(1,1), the NN-GP-GM(1,1), and the proposed models have something in common. It is evident that they are grey residual modification models and developed for residual sign estimation to improve the prediction accuracy of the residual modification model. However, it is interesting to estimate not only the sign but the extent to which $\hat{x}_{k}^{(0)}$ obtained from the original $\operatorname{GM}(1,1)$ model can be modified by $\hat{\varepsilon}_{k}^{(0)}(k=2,3, \ldots, n)$. This remains for the future work.

Acknowledgments-The authors would like to thank the anonymous referees for their valuable comments. This research is partially supported by the Ministry of Science and Technology, Taiwan, under Grant MOST 104-2410-H-033-023-MY2.

\section{References}

Chang CJ, Dai WL and Chen CC (2015). A novel procedure for multimodel development using the grey silhouette coefficient for small-data-set forecasting. Journal of the Operational Research Society 66(11):1887-1894.

Chang CJ, Li DC, Huang YH and Chen CC (2015). A novel gray forecasting model based on the box plot for small manufacturing data sets. Applied Mathematics and Computation 265(C):400-408.

Cui J, Liu SF, Zeng B and Xie NM (2013). A novel grey forecasting model and its optimization. Applied Mathematical Modelling 37(6):4399-4406.

Deng JL (1982). Control problems of grey systems. Systems and Control Letters 1(5):288-294.

Duran Toksari M (2009). Estimating the net electricity energy generation and demand using ant colony optimization approach: Case of Turkey. Energy Policy 37(3):1181-1187.

Ediger VS and Akar S (2007). ARIMA forecasting of primary energy demand by fuel in Turkey. Energy Policy 35(3):1701-1708.

Feng SJ, Ma YD, Song ZL and Ying J (2012). Forecasting the energy consumption of China by the grey prediction model. Energy Sources, Part B: Economics, Planning, and Policy 7(4):376-389.

Goldberg DE (1989). Genetic Algorithms in Search, Optimization, and Machine Learning. MA: Addison-Wesley.

Gonzalez PA and Zamarreno JA (2005). Prediction of hourly energy consumption in buildings based on a feedback artificial neural network. Energy and Buildings 37(6):595-601.

Hsu CC and Chen CY (2003). Applications of improved grey prediction model for power demand forecasting. Energy Conversion and Management 44(14):2241-2249.

Hsu CI and Wen YU (1998). Improved grey prediction models for trans-Pacific air passenger market. Transportation Planning and Technology 22(2):87-107.

$\mathrm{Hu}$ YC (2010). Pattern classification by multi-layer perceptron using fuzzy integral-based activation function. Applied Soft Computing 10(3):813-819.

$\mathrm{Hu}$ YC (2016). Pattern classification using grey tolerance rough sets. Kybernetes 45(2):266-281.

Hu YC, Tzeng GH, Hsu YT and Chen RS (2001). Using learning algorithm to find the developing coefficient and control variable of $\operatorname{GM}(1,1)$ model. Journal of the Chinese Grey System Association 4(1):17-26.

$\mathrm{Hu}$ YC, Chiu YJ, Liao YL and Li Q (2015). A fuzzy similarity measure for collaborative filtering using nonadditive grey relational analysis. Journal of Grey System 27(2):93-103. 
Lauret P, Fock E, Randrianarivony RN and Manicom-Ramasamy JF (2008). Bayesian neural network approach to short time load forecasting. Energy Conversion and Management 49(5): 1156-1166.

Lee SC and Shih LH (2011). Forecasting of electricity costs based on an enhanced gray-based learning model: A case study of renewable energy in Taiwan. Technological Forecasting and Social Change 78(7): 1242-1253.

Lee YS and Tong LI (2011). Forecasting energy consumption using a grey model improved by incorporating genetic programming. Energy Conversion and Management 52(1):147-152.

Lewis C (1982). Industrial and Business Forecasting Methods. London: Butterworth Scientific.

Liu ZY (2015). Global Energy Internet. Beijing: China Electric Power Press.

Liu S and Lin Y (2006). Grey Information: Theory and Practical Applications. London: Springer.

Luo DR, Guo KZ and Huang HR (2013). Regional economic forecasting combination model based on RAR + SVR. In: Cao $\mathrm{BY}$ and Nasseri $\mathrm{H}$ (Eds) Fuzzy Information \& Engineering and Operations Research \& Management, Advances in Intelligent Systems and Computing, vol. 211. Berlin: Springer, pp. 329-338.

Makridakis S (1993). Accuracy measures: Theoretical and practical concerns. International Journal of Forecasting 9(4):527-529.

ManKF, Tang KS and Kwong S (1999). Genetic Algorithms: Concepts and Designs. London: Springer.

Mao MZ and Chirwa EC (2006). Application of grey model GM(1,1) to vehicle fatality risk estimation. Technological Forecasting and Social Change 73(5):588-605.

National Bureau of Statistics of China (2014). China Statistical Yearbook 2014. Beijing: China Statistics Press.

Pi D, Liu J and Qin X (2010). A grey prediction approach to forecasting energy demand in China. Energy Sources, Part A: Recovery, Utilization, and Environmental Effects 32(16):1517-1528.

Rooij AJF, Jain LC and Johnson RP (1996). Neural Network Training Using Genetic Algorithms. Singapore: World Scientific.

Smith KA and Gupta JND (2002). Neural Networks in Business: Techniques and Applications. Hershey, PA: Idea Group.

Suganthi L and Samuel AA (2012). Energy models for demand forecasting-A review. Renewable and Sustainable Energy Reviews 16(2):1223-1240.

Tsaur RC and Liao YC (2007). Forecasting LCD TV demand using the fuzzy grey model $\operatorname{GM}(1,1)$. International Journal of Uncertainty, Fuzziness and Knowledge-Based Systems 15(6):753-767.
Tutun S, Chou CA and Canıyılmaz E (2015). A new forecasting framework for volatile behavior in net electricity consumption: A case study in Turkey. Energy 93(2):2406-2422.

Vonkj E, Jain LC and Johnson RP (1997). Automatic Generation of Neural Network Architecture Using Evolutionary Computation. Singapore: World Scientific.

Wang ZX and Hao P (2016). An improved grey multivariable model for predicting industrial energy consumption in China. Applied Mathematical Modelling 40(11-12):5745-5758.

Wang CH and Hsu LC (2008). Using genetic algorithms grey theory to forecast high technology industrial output. Applied Mathematics and Computation 195(1):256-263.

Wei J, Zhou L, Wang F and Wu D (2015). Work safety evaluation in Mainland China using grey theory. Applied Mathematical Modelling 39(2):924-933.

Weiss SM and Kulikowski CA (1991). Computer Systems That Learn: Classification and Prediction Methods from Statistics, Neural Nets, Machine Learning, and Expert Systems. CA: Morgan Kaufmann.

Wen KL (2004). Grey Systems Modeling and Prediction. Tucson: Yang's Scientific Research Institute.

Wu L, Liu S and Wang Y (2012). Grey Lotka-Volterra model and its application. Technological Forecasting and Social Change 79(9): 1720-1730.

Wu L, Liu S, Yao L, Yan S and Liu D (2013). Grey system model with the fractional order accumulation. Communications in Nonlinear Science and Numerical Simulation 18(7):1775-1785.

Wu L, Liu S, Yao L, Yan S and Liu D (2013). The effect of sample size on the grey system model. Applied Mathematical Modelling 37(9):6577-6583.

Xia C, Wang J and ShortMcMenemy K (2010). Medium and long term load forecasting model and virtual load forecaster based on radial basis function neural networks. Electrical Power and Energy Systems 32(7):743-750.

Xie NM and Liu SF (2009). Discrete grey forecasting model and its optimization. Applied Mathematical Modelling 33(2):1173-1186.

Zeng B, Meng W and Tong MY (2016). A self-adaptive intelligence grey predictive model with alterable structure and its application. Engineering Applications of Artificial Intelligence 50(C):236-244.

Zhou P, Ang BW and Poh KL (2006). A trigonometric grey prediction approach to forecasting electricity demand. Energy 31(14):2839-2847.

Received 18 April 2016; accepted 13 September 2016 